\title{
First order optical differentiator based on an FBG in transmission
}

\author{
Miguel A. Preciado*, Xuewen Shu, Paul Harper, and Kate Sugden \\ Aston Institute of Photonic Technologies, Aston University, Birmingham, B4 7ET, UK. \\ *Corresponding author: m.preciado@aston.ac.uk
}

Optical differentiators constitute a basic device for analog all-optical signal processing [1]. Fiber grating approaches, both fiber Bragg grating (FBG) and long period grating (LPG), constitute an attractive solution because of their low cost, low insertion losses, and full compatibility with fiber optic systems. A first order differentiator LPG approach was proposed and demonstrated in [2], but FBGs may be preferred in applications with a bandwidth up to few $\mathrm{nm}$ because of the extreme sensitivity of LPGs to environmental fluctuations [3]. Several FBG approaches have been proposed in [3-6], requiring one or more additional optical elements to create a first-order differentiator. A very simple, single optical element FBG approach was proposed in [7] for first order differentiation, applying the well-known logarithmic Hilbert transform relation of the amplitude and phase of an FBG in transmission [8]. Using this relationship in the design process, it was theoretically and numerically demonstrated that a single FBG in transmission can be designed to simultaneously approach the amplitude and phase of a first-order differentiator spectral response, without need of any additional elements.

In this paper we experimentally demonstrate a first-order optical differentiator based on an FBG in transmission using the ideas introduced in [7]. In order to prove the concept, we characterized the FBG with an optical vector analyser, and performed an optical pulse differentiation experiment where the signals were characterized using an optical spectrum analyser (OSA) and a second harmonic generation (SHG) frequency resolved optical grating (FROG) system. We show that the resulting differentiated pulse is in very good agreement with the numerically predicted differentiated pulse, validating this simple and inexpensive solution for the fabrication of first-order optical differentiators.
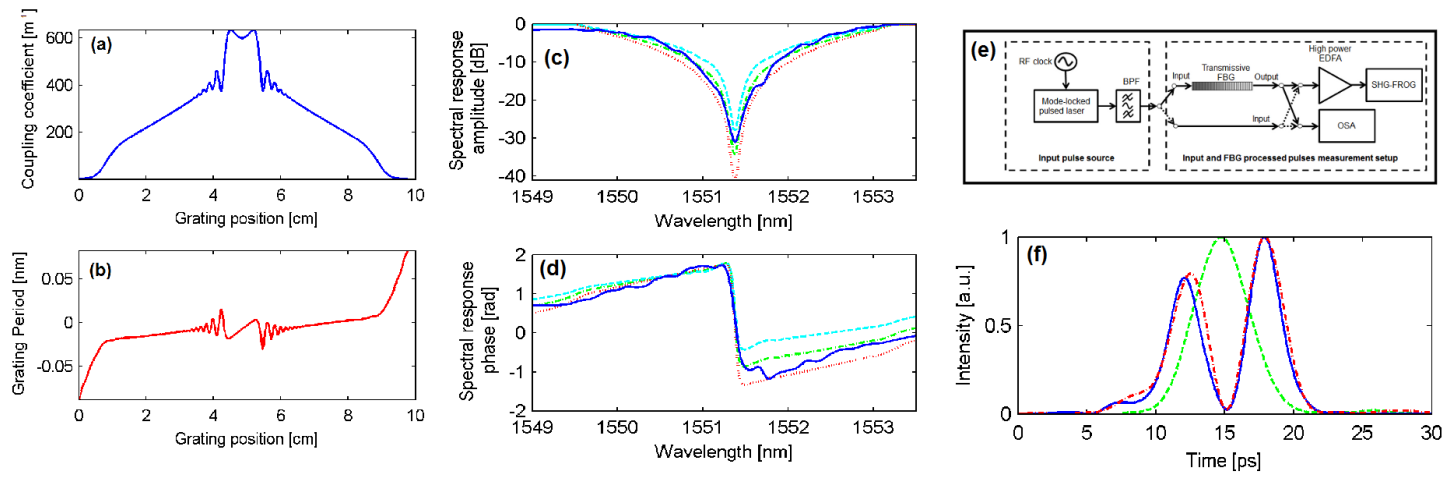

Fig. 1. Coupling coefficient (a), and grating period (b) of the designed FBG obtained from inverse scattering; (c) and (d) show a comparison between the spectral response in transmission (c),(d) of the experimentally characterized FBG (solid blue), and the simulation results using the designed coupling coefficient $k(z)$ (dotted red), and the coupling coefficient with a $10 \%$ variation $0.9 \cdot(z)($ dash-dotted green), and a $20 \%$ variation $0.8 \mathrm{k}(\mathrm{z})$ (dashed cyan); (e) experimental setup used to prove the operation of the fabricated FBG in transmission differentiator. BPF, bandpass filter; EDFA, amplifier; ( $f$ ) FROG recovered temporal intensity of the input pulse (dashed green), output pulse (solid blue), and numerically calculated temporal differentiation (dash-dotted red).

\section{References}

1. N. Q. Ngo and L. N. Binh, "Theory of a FIR optical digital differentiator," Fiber and Integrated Opt., 14, 359-385 (1995)

2. R. Slavík, Y. Park, M. Kulishov, R. Morandotti, and J. Azaña, "Ultrafast all-optical differentiators," Opt. Express 14, 10699-10707 (2006)

3. N. K. Berger, B. Levit, B. Fischer, M. Kulishov, D. V. Plant, and J. Azaña, "Temporal differentiation of optical signals using a phaseshifted fiber Bragg grating,” Opt. Express 15, 371-381 (2007).

4. L.-M. Rivas, K. Singh, A. Carballar, and J. Azaña, "Arbitrary-order ultra-broadband all-optical differentiators based on fiber Bragg gratings," IEEE Photon. Technol. Lett. 19, 1209-1211 (2007)

5. M. A. Preciado, V. Garcia-Muñoz, and M. A. Muriel, "Ultrafast all-optical Nth-order differentiator based on chirped fiber Bragg gratings," Opt. Express 15, 7196-7201 (2007).

6. M. Li, D. Janner, J. Yao, and V. Pruneri, "Arbitrary-order all-fiber temporal differentiator based on a fiber Bragg grating: design and experimental demonstration," Opt. Express 17, 19798-19807 (2009)

7. M. A. Preciado and M. A. Muriel, "Design of an ultrafast all-optical differentiator based on a fiber Bragg grating in transmission," Opt. Lett. 33, 2458-2460 (2008).

8. J. Skaar, "Synthesis of fiber Bragg gratings for use in transmission," J. Opt. Soc. Am. A 18, 557-564 (2001). 